\title{
Checklist de Siphonaptera (Insecta) do Estado de São Paulo
}

\author{
Pedro Marcos Linardi ${ }^{1,2}$ \\ ${ }^{1}$ Departamento de Parasitologia, Instituto de Ciências Biológicas, \\ Universidade Federal de Minas Gerais - UFMG, \\ Av. Antônio Carlos, n. 6627, CEP 31270-901, Belo Horizonte, MG, Brasil \\ ${ }^{2}$ Autor para correspondência: Pedro Marcos Linardi, e-mail: linardi@icb.ufmg.br
}

LINARDI, P.M. Checklist of Siphonaptera (Insecta) from São Paulo State, Brazil. Biota Neotrop. 11(1a): http://www.biotaneotropica.org.br/v11n1a/en/abstract?inventory+bn0301101a2011.

\begin{abstract}
Adult fleas are haematophagous insects that act as infesting agents or as vectors of pathogens. Your hosts are endothermic animals, essentially rodents. The flea fauna of São Paulo State represents 61.3\% (38/62) of the Brazilian's fauna, being the richest when compared with other States in number of species or genera. Some species are known only by one or other of the sexes; others, through limited numbers of specimens collected. Four species are endemic (10.5\%) in the State. In Brazil, the main collection of fleas is deposited at the Museum of Zoology of USP.
\end{abstract}

Keywords: Siphonaptera, biodiversity of the State of São Paulo, BIOTA/FAPESP Program.

Number of species: In the world: 4,000, in Brazil: 100, estimated in São Paulo State: 50.

LINARDI, P.M. Checklist dos Siphonaptera (Insecta) do Estado de São Paulo, Brasil. Biota Neotrop. 11(1a): http://www.biotaneotropica.org.br/v11n1a/pt/abstract?inventory+bn0301101a2011.

Resumo: As pulgas são insetos hematófagos na fase adulta e que atuam como agentes infestantes ou como vetores de patógenos. Seus hospedeiros são animais endotérmicos, essencialmente mamíferos e, predominantemente roedores. A sifonapterofauna do Estado de São Paulo representa 61,3\% (38/62) da brasileira, sendo a mais rica quando comparada com outros estados em número de espécies ou gêneros. Algumas espécies são conhecidas apenas por um dos sexos; outras, através de pequeno número de exemplares colecionados. Quatro espécies (10,5\%) são endêmicas no Estado. No Brasil, a principal coleção de pulgas está depositada no Museu de Zoologia da USP. Palavras-chave: Siphonaptera, biota paulista, Programa BIOTA/FAPESP.

Número de espécies: No mundo: 4.000, no Brasil: 100, estimadas no estado de São Paulo: 50. 


\section{Introdução}

A ordem Siphonaptera compreende insetos ápteros, pequenos (2,0-3,0 $\mathrm{mm}$ em média), de coloração castanha, achatados lateralmente, com as pernas posteriores adaptadas para o salto e o corpo revestido por cerdas dirigidas para trás. A maior parte das espécies conhecidas apresenta ctenídios (= pentes) que são cerdas mais robustas e esclerosadas destinadas à fixação e locomoção das pulgas entre os pêlos dos hospedeiros.

Vulgarmente esses insetos são conhecidos como pulgas ("flea", "puce”, "pulce”, "flöh”, "blokha" "pulex", "psyllos" em outros idiomas) e bichos-do-pé, estes também denominados tom, sico, tunga, pulga da areia, jatecuba, bicho-do-porco, etc (Cunha 1914).

O dimorfismo sexual é acentuado, com as fêmeas maiores que os machos e apresentando a parte posterior arredondada. Os machos, pelo fato de albergarem o aparelho copulador nos últimos segmentos, apresentam a extremidade posterior voltada para cima. $\mathrm{O}$ aparelho bucal é do tipo sugador-pungitivo apenas na fase adulta, com o repasto sanguíneo exercido pelos dois sexos, ainda que nas fêmeas ele também seja importante para a maturação ovariana e posterior oviposição. A metamorfose é completa (holometábolos), os ovos são esbranquiçados, ovóides ou elipsoidais (300-700 $\mu \mathrm{m}$ em média) e as larvas são vermiformes, esbranquiçadas, ápodas, com aparelho bucal mastigador.

O ciclo biológico, de ovo a adulto, completa-se em aproximadamente 25-30 dias, dependendo das condições de temperatura, umidade e alimentação obtida pelas larvas, com a emergência das fêmeas, em algumas espécies antecedendo a dos machos (Linardi \& Nagem 1972). A eclosão ocorre dentro de um a dois dias, 24-36 horas após o primeiro repasto sanguíneo. Há três estádios larvários, cada um deles passando por mudas a cada três dias; exceção para os tungídeos, com apenas dois instares larvários (Hicks 1930, Barnes \& Radovsky 1969). As pulgas alimentam-se diretamente sobre os capilares (solenófagas), cada repasto durando cerca de 10-15 minutos. Na maioria das espécies a hematofagia persiste, mesmo após as fêmeas estarem repletas, uma vez que o sangue digerido e posteriormente eliminado pelo ânus é fundamental para a nutrição e desenvolvimento das larvas.

Embora possam locomover-se lentamente pelo ambiente e entre os pelos dos hospedeiros, o salto é o principal recurso utilizado para alcançarem seus hospedeiros, podendo chegar até $33 \mathrm{~cm}$ em algumas espécies e em ritmo de até 600 pulos por hora (Rothschild et al. 1973).

Esses insetos têm uma história evolutiva de 60 milhões de anos, sendo já encontrados em mamíferos pré-históricos. Os hospedeiros são animais endotérmicos, especialmente mamíferos (94\%). Segundo Marshall (1981), os seguintes percentuais de infestação são encontrados entre as ordens de mamíferos: Rodentia (74\%), Insectivora (8\%), Marsupialia (= Didelphimorphia 5\%), Chiroptera (5\%), Lagomorpha (3\%), Carnivora (3\%). Menos de $1 \%$ dos registros são encontrados em Monotremata, Xenarthra (Cingulata + Pilosa), Pholidota, Hyracoidea e Artiodactyla. Em Primates, apenas o homem é tido como hospedeiro habitual. A ocorrência em répteis é acidental. A ordem Rodentia é a mais importante porque contém o maior número de espécies parasitadas, além de epidemiologicamente algumas destas espécies funcionarem como reservatórios de infecções transmitidas por pulgas (peste, tifo murino, tularemia) e, ecologicamente, ocuparem diversos nichos em diferentes ecótopos. Para Traub (1980), os hospedeiros primitivos tendem ser mais infestados por pulgas e piolhos primitivos, ao contrário dos mais evoluídos associados com ectoparasitos mais recentes.

Relativamente à permanência no hospedeiro, três modalidades de parasitismo são observadas: 1) pulgas penetrantes, cujas fêmeas introduzem a cabeça, tórax e parte do abdome nos hospedeiros, lá permanecendo até a expulsão dos últimos ovos, quando então se atrofiam, murchando-se, e.g. Tunga penetrans (L., 1758), ou semi-penetrantes, em que penetram apenas parte da cabeça nos hospedeiros, deixando o tórax e o abdome a descoberto (Hectopsylla spp.); 2) pulgas, cujos adultos vivem e permanecem sobre os respectivos hospedeiros (a maioria das espécies: Xenopsylla spp., Ctenocephalides spp., Polygenis spp.); 3) aquelas nas quais os adultos não permanecem sobre os hospedeiros, só os procurando para a hematofagia: Pulex irritans L., 1758 e pulgas de aves.

Independentemente do parasitismo temporário ou permanente, as pulgas podem ser específicas ou ecléticas em relação à preferência alimentar. Aproximadamente, 600 espécies de pulgas são específicas, cada uma delas infestando um único hospedeiro, sendo conhecidas através de um único registro (Medvedev 2002). As associações específicas, caracterizadas pela exclusividade de hospedeiros constituem um meio auxiliar para a identificação taxonômica dos respectivos hospedeiros, podendo subsidiar mastozoologistas (Linardi 1977). O ecletismo de certas espécies, em razão da diversidade de hospedeiros e poli-hematofiagismo, é um parâmetro importante no estudo de questões epidemiológicas relativas à transmissão de certos patógenos. O intercâmbio de pulgas entre roedores silvestres e sinantrópicos pode revelar proximidade de faunas, estreitando o fluxo de patógenos entre os vetores. Ainda que para Traub (1980), a especificidade seja mais regra que exceção, do ponto de vista parasitológico e/ou epidemiológico, o ecletismo é fundamental, em virtude da troca de hospedeiros e a veiculação de moléstias. Neste sentido, as pulgas atuam em duas vertentes: 1) como agentes infestantes ou parasitos propriamente ditos; 2) como vetoras de agentes infecciosos. No primeiro caso são diretamente responsáveis por: a) ações irritativas, provocando pruridos e outros sintomas clínicos conhecidos como dermatites alérgicas, comuns em cães e gatos e prurigo de Hebra, ocorrendo no homem (Almeida \& Croce 1990); b) ações espoliadoras, em função de altas infestações em animais de pequeno porte podendo conduzir à anemia, já que as pulgas picam os hospedeiros várias vezes ao dia, ingerindo quantidades de sangue que aumentam, significativamente, o seu peso corpóreo; c) ações inflamatórias produzidas por fêmeas de pulgas penetrantes (tungíase), cujos orifícios deixados no corpo dos respectivos hospedeiros tornam-se passíveis de infecção por agentes oportunistas, podendo provocar tétano, gangrena gasosa e blastomicoses.

Como vetoras, ou hospedeiras intermediárias, as pulgas são incriminadas na transmissão de viroses (mixomatose), doenças bacterianas (tifo murino, bartonelose, salmoneloses, tularemia, peste), protozooses (tripanossomíases) e helmintoses (himenolepíases, dilepidiose, filarioses, infecções por tilenquídeos), bem como podem ser infectadas ou infestadas por outros artrópodos. Ainda que a peste seja, sem dúvida, a principal moléstia transmitida pelas pulgas, dada a sua morbidade, letalidade e registro histórico, tendo dizimado $1 / 4 \mathrm{da}$ população européia no século XIX, cumpre salientar que ela foi introduzida no Brasil em 1899 pelo porto de Santos, daí se expandiu para outras cidades através do tráfego comercial e ferrovias, estando hoje assentada na zona rural em habitat natural, correspondendo a uma área de $240.000 \mathrm{~km}^{2}$ e ocorrendo em 971 localidadesfocos, situadas em 189 municípios brasileiros. Os roedores são os reservatórios da infecção, sejam eles sinantrópicos ou silvestres. Se bem que em todo o mundo mais de 200 espécies de pulgas possam potencialmente transmitir a peste e que mais de 20 espécies piquem facilmente o homem, Xenopsylla cheopis (Rothschild, 1903), Xenopsylla brasiliensis (Baker, 1904) e as espécies de Polygenis desempenham papel relevante no Brasil. Os pulicídeos, essencialmente $X$. cheopis, são responsáveis pela transmissão de Yersinia pestis dos roedores sinantrópicos para o homem, enquanto que outras espécies, especialmente as contidas no gênero Polygenis, 
mantêm a peste entre roedores silvestres ou entre roedores silvestres e domiciliares. Relativamente às riquetsioses, há registros de tifo murino em São Paulo: o agente etiológico é a Rickettsia typhi (= R. mooseri) e os reservatórios são roedores sinantrópicos, com a transmissão do patógeno sendo realizada pelas fezes de $X$. cheopis. Recentemente, Horta et al. (2007) diagnosticaram Rickettsia felis em Ctenocephalides felis felis (Bouché, 1835) capturadas de cães e gatos e em Polygenis (Neopolygenis) atopus (Jordan \& Rothschild, 1922) coletadas de gambás, em cinco áreas do estado de São Paulo. A técnica da PCR tem também permitido reconhecer DNA de Leishmania chagasi em C. felis felis retiradas de cães naturalmente infectados, abrindo assim a possibilidade da transmissão mecânica do calazar canino por meio de pulgas (Coutinho 2003, Coutinho \& Linardi 2007). Recentemente, Ferreira et al. (2009) usando a mesma técnica em pulgas de cães de Araçatuba/SP chegaram às mesmas conclusões de Coutinho \& Linardi (2007). Conforme observado por Pacheco et al. (1998), os tripanosomatídeos monoxênicos de pulgas podem causar infecções oportunistas em indivíduos imunodeficientes.

Atualmente são conhecidas cerca de 3.000 espécies, grupadas em 240 gêneros (ou 330 quando incluindo subgêneros), 44 tribos, 28 famílias e 15 famílias (Lewis 1998), distribuindo-se da região Ártica até a Antártica. Outros autores, entre eles Linardi \& Guimarães (2000), consideram Tungidae como família distinta, somando-se assim 16. Para Medvedev (1994), as famílias seriam 18, incluídas em quatro infra-ordens. As regiões temperadas são as mais ricas em espécies de pulgas, tanto latitudinal como longitudinalmente. Para Wenzel \& Tipton (1966), isto se deve à maior preferência dos roedores pelos climas mais frios. A distribuição geográfica das espécies de pulgas está provavelmente relacionada com deriva continental e placas tectônicas e subseqüente dispersão e redistribuição dos táxons hospedeiros (Traub 1980). Excetuandose algumas formas introduzidas e considerando a Europa e Ásia como um todo - Eurásia - nenhum gênero de pulga de mamífero é encontrado em quatro ou mais continentes; apenas dois ocorrem em três continentes, entre eles, Tunga. A sifonapterofauna da região Paleártica é a mais diversificada, representando 38\% do número total de espécies conhecidas, com o restante distribuído similarmente entre as demais regiões geográficas. Por outro lado, a percentagem de gêneros endêmicos chega a $61 \%$ na região Afrotropical, seguida pela Australiana (58\%), Neotropical (56\%), Paleártica (45\%), Oriental (42\%) e Neártica (37\%)(Krasnov 2008).

Ao todo, 223 espécies têm sido relacionadas como hospedeiras para pulgas no Brasil, das quais 115 (51,6\%) são de roedores (Linardi \& Guimarães 2000). Outros grupos de hospedeiros apresentam os seguintes percentuais de registros: Carnivora (13,0\%); Didelphimorphia (13,0\%); Chiroptera (8,1\%); Aves (5,4\%); Xenarthra (3,6\%); Primates (2,7\%); Artiodactyla (1,8\%); Lagomorpha e Perissodactyla $(0,4 \%)$. Dados complementares sobre hospedeiros de sifonápteros ropalopsilinos - incluídos na mais importante família de pulgas do Brasil (Rhopalopsyllidae) - podem ser encontrados em Linardi (1985a), ao indicar para cada uma das 77 espécies e/ou subespécies de pulgas, os principais gêneros de hospedeiros e os índices de registro de infestação pelos respectivos sifonápteros.

\section{Metodologia}

\section{Lista das fontes consultadas para elaboração da lista}

Para a elaboração da lista das espécies de sifonápteros assinaladas no estado de São Paulo, as seguintes fontes foram consultadas: Lutz
(1903), Cunha (1914), Pinto (1925, 1930, 1931), Pinto \& Dreyfus (1927), Fonseca \& Prado (1932), Meira (1931, 1932, 1934), Pessoa \& Horta (1933), Prado (1933), Guimarães (1936a, 1936b, 1938, 1940, 1942, 1944, 1945, 1947, 1948, 1954), Lima (1940, 1943), Lima \& Hathaway (1946), Vaz \& Rocha (1946), Hopkins \& Rothschild (1953, 1956, 1962, 1966, 1971), Johnson (1957), Machado-Allison (1962), Linardi (1974, 1977, 1978, 1979, 1981a, b, 1984a, b, c, 1985a, b, 1987a, b, 1999), Nagem (1977), Smit (1987), Linardi \& Guimarães (1993, 2000), Guimarães \& Linardi (1993), Bossi (1996, 2003), Barros-Battesti et al. (1997), Bossi et al. (2002), Mascarenhas (2002) Moraes et al. (2003), Linardi \& Lareschi (2003), Nieri-Bastos et al. (2003, 2004a, 2004b), San'Anna et al. (2004), Horta (2006), Horta et al. (2007), Avelar (2010).

A nomenclatura utilizada segue Linardi \& Guimarães (2000).

\section{Resultados \& Discussão}

As espécies já assinaladas no Estado de São Paulo estão indicadas no checklist (modelo à parte).

Relativamente à ocorrência de Tunga trimamillata Pampiglione et al., 2002, trata-se de novo registro, já que anteriormente, Vaz \& Rocha (1946) relataram diferenças morfológicas entre T. penetrans e o material colhido de bovinos de Barretos, SP. Esta espécie está sendo agora constatada por Avelar (2010) em exemplares coletados no estado do Rio de Janeiro. Como registro acidental, o de Meira (1934), quando encontrou um único exemplar de Synosternus pallidus (Taschenberg, 1880), entre 13.431 pulgas capturadas de 1.404 roedores urbanos em São Paulo, SP. De fato, essa espécie não deve ter se estabelecido no Brasil, já que decorridos mais de 75 anos, nenhum outro registro foi informado. Entre as espécies já assinaladas no estado, apenas cinco $(13,1 \%)$ foram descritas nos últimos 60 anos, com a maioria tendo sido conhecida entre $1901 \mathrm{e}$ 1950: $65,8 \%$ (25).

As principais sinonímias e outros dados taxonômicos relativos às espécies que ocorrem em São Paulo são apresentados na Tabela 1. Observando-se os nomes específicos válidos e os das respectivas sinonímias nota-se que nove deles foram dados em homenagens a eminentes entomologistas ou parasitologistas pátrios, ou mesmo aos respectivos colecionadores: Adolfo Lutz, Lauro Pereira Travassos, Alcides Prado, Roberto de Almeida Cunha, Antônio Ronna, Lindolpho Rocha Guimarães, Carlos Lako, Emílio Dente e Hugo de Souza Lopes, quatro desses paulistas (Prado, Guimarães, Lako, Dente) e outros dois (Lutz e Travassos) com brilhantes atuações, quando de suas passagens, respectivamente, pelo Instituto de Bacteriologia (mais tarde, Instituto Adolfo Lutz) e Faculdade de Medicina de São Paulo. Posteriormente, Guimarães (1956) ainda homenagearia o notável Samuel Barnsley Pessôa ao nomear uma espécie, endêmica em Pernambuco, como Polygenis pessoai, atualmente, Polygenis (P.) axius pessoai Guimarães, 1956.

Salvo poucas exceções, a maioria das espécies exibe a proporção entre os sexos próxima a 1,00. Via de regra, as fêmeas são também maiores que os machos (Tabela 1). As dimensões das fêmeas ingurgitadas (neosomas) constituem caráter diagnóstico auxiliar para as espécies de Tunga. Entre as espécies listadas, 10 (26,3\%) foram descritas do estado de São Paulo.

Os hospedeiros e a distribuição geográfica dessas mesmas espécies estão indicados na Tabela 2. A maioria dos hospedeiros pertence à ordem Rodentia $(63,2 \%)$, sejam eles silvestres (especialmente, Akodon, Oryzomys), comensais ou domiciliares (Rattus e Mus). 
Linardi, P.M

Tabela 1. Dados taxonômicos e morfométricos das espécies de Siphonaptera do Estado de São Paulo, Brasil.

Table 1. Taxonomic and morphometric data of the species of Siphonaptera from São Paulo State, Brazil.

\begin{tabular}{|c|c|c|c|c|c|}
\hline $\mathbf{N}^{\circ}$. & Espécies & $\begin{array}{c}\text { Vicissitudes } \\
\text { nomenclaturais }\end{array}$ & Distribuição dos sexos & $\begin{array}{c}\text { Dimensões médias } \\
(\mathbf{m m})\end{array}$ & Observações \\
\hline 1 & Pulex irritans & $\begin{array}{c}\text { Pulex ater, Pulex irritans } \\
\text { var. bahiensis, Pulex } \\
\text { conepati }\end{array}$ & Relação $+/ \delta^{\Uparrow}: 1,00$ & 1 $1,80+2,32$ & - \\
\hline 2 & Xenopsylla cheopis & $\begin{array}{c}\text { Pulex cheopis, Loemopsylla } \\
\text { cheopis }\end{array}$ & Relação $+/ \delta^{\Uparrow}: 1,00$ & $\lambda 1,55+2,40$ & - \\
\hline 3 & Xenopsylla brasiliensis & $\begin{array}{l}\text { Pulex brasiliensis, } \\
\text { Loemopsylla vigetus }\end{array}$ & Relação $+/ \delta^{\Uparrow}: 1,50$ & त̂ 1,86 ○ 2,28 & - \\
\hline 4 & Ctenocephalides canis & $\begin{array}{c}\text { Pulex canis, Ctenocephalus } \\
\text { canis }\end{array}$ & Relação $+/ \delta: 2,40$ & त 1,94 ㅇ 2,70 & - \\
\hline 5 & Ctenocephalides felis felis & $\begin{array}{l}\text { Pulex felis, Ctenocephalus } \\
\text { felis }\end{array}$ & Relação $/$ / & 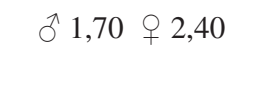 & - \\
\hline 6 & Tunga penetrans & $\begin{array}{c}\text { Pulex penetrans, } \\
\text { Dermatophilus penetrans }\end{array}$ & Relação $+/ \delta$ : 1,00 & $\begin{array}{l}\text { o } 1,05+1,00 \\
\text { neosoma } 6 \times 5\end{array}$ & - \\
\hline 7 & Tunga caecata & $\begin{array}{l}\text { Sarcopsylla caecata, } \\
\text { Dermatophilus caecata }\end{array}$ & Machos desconhecidos & neosoma $7 x 6$ & $\begin{array}{c}\text { Espécie descrita do Brasil: } \\
\text { São Paulo }\end{array}$ \\
\hline 8 & Tunga travassosi & - & Machos desconhecidos & neosoma $13 \times 10$ & $\begin{array}{c}\text { Espécie descrita do Brasil: } \\
\text { São Paulo }\end{array}$ \\
\hline 9 & Tunga bondari & - & Machos desconhecidos & neosoma $6 \times 6$ & $\begin{array}{c}\text { Espécie descrita do Brasil: } \\
\text { Bahia }\end{array}$ \\
\hline 10 & Tunga terasma & - & - & neosoma $10 \times 8$ & $\begin{array}{c}\text { Espécie descrita do Brasil: } \\
\text { Goiás }\end{array}$ \\
\hline 11 & Tunga trimamillata & - & - & $\begin{array}{l}\text { 1 } 1,11 \text { q } 1,20 \\
\text { neosoma } 12 \times 5\end{array}$ & - \\
\hline 12 & Hectopsylla psittaci & Pulex (Hectopsylla) testudo & - & ○ 2,38 & - \\
\hline 13 & Hectopsylla pulex & Rhynchopsyllus pulex & $\begin{array}{l}\text { Machos apenas em } \\
\text { guano de morcego }\end{array}$ & $+2,50$ & Espécie descrita do Brasil \\
\hline 14 & Nosopsyllus (N.) fasciatus & $\begin{array}{c}\text { Pulex fasciatus, } \\
\text { Ceratophyllus fasciatus }\end{array}$ & Relação + ( $/{ }^{\prime}: 2,00$ & + 2,72 & - \\
\hline 15 & Leptopsylla (L.) segnis & $\begin{array}{c}\text { Pulex segnis, Ctenopsyllus } \\
\text { musculi }\end{array}$ & Relação $+/ \delta$ : 1,84 & ô 1,65 o 1,95 & - \\
\hline 16 & Hormopsylla fosteri & $\begin{array}{l}\text { Ceratophylla fosteri, } \\
\text { Hexactenopsylla fosteri }\end{array}$ & Relação $+/ \widehat{O}: 2,00$ & of 2,10 ㅇ 2,43 & - \\
\hline 17 & $\begin{array}{l}\text { Craneopsylla minerva } \\
\text { minerva }\end{array}$ & Stephanocircus minerva & Relação $+/ \widehat{~}: 2,48$ & of 2,44 q 3,00 & - \\
\hline 18 & $\begin{array}{l}\text { Adoratopsylla (A.) a. } \\
\text { antiquorum }\end{array}$ & $\begin{array}{l}\text { Spalocopsylla antiquorum, } \\
\text { Stenopsylla cunhai }\end{array}$ & Relação $+/ \delta$ : 1,10 & $2,33 q 2,77$ & - \\
\hline 19 & $\begin{array}{l}\text { Adoratopsylla (A.) a. } \\
\text { ronnai }\end{array}$ & - & - & - & $\begin{array}{c}\text { Espécie descrita do Brasil: } \\
\text { Rio G. do Sul }\end{array}$ \\
\hline 20 & $\begin{array}{l}\text { A. }(\text { Tritopsylla }) i . \\
\text { intermedia }\end{array}$ & $\begin{array}{l}\text { Doratopsylla intermedia, } \\
\text { Tritopsylla } i \text {. intermedia }\end{array}$ & Relação $+/ \delta$ : 0,95 & 今 2,73 ㅇ 3,34 & - \\
\hline 21 & Rhopalopsyllus lutzi lutzi & Pulex lutzi & Relação $+/ \widehat{o}: 1,41$ & o $2,50+2,92$ & $\begin{array}{c}\text { Espécie descrita do Brasil: } \\
\text { São Paulo }\end{array}$ \\
\hline 22 & $\begin{array}{l}\text { Rhopalopsyllus lugubris } \\
\text { lugubris }\end{array}$ & Rhopalopsyllus bohlsi & Relação $+/ \widehat{\jmath}: 2,76$ & 今 2,56 ㅇ 3,11 & - \\
\hline 23 & $\begin{array}{l}\text { Rhopalopsyllus australis } \\
\text { tamoyus }\end{array}$ & - & Relação $+/ \delta$ : 3,09 & j 1,82 ㅇ 2,28 & - \\
\hline 24 & R. australis tupiniquinus & - & $\begin{array}{c}\text { Apenas } 3 \text { exemplares } \\
\text { conhecidos }\end{array}$ & - & $\begin{array}{c}\text { Espécie descrita do Brasil: } \\
\text { São Paulo }\end{array}$ \\
\hline 25 & Hechtiella lakoi & $\begin{array}{l}\text { Polygenis lakoi, Tiamastus } \\
\text { (Hechtiella) lakoi }\end{array}$ & Relação $+/ \delta: 2,14$ & 今 1,77 ㅇ 2,40 & $\begin{array}{c}\text { Espécie descrita do Brasil: } \\
\text { Espírito Santo }\end{array}$ \\
\hline 26 & Hechtiella lopesi & - & 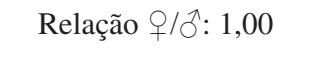 & 2,18 웅 2,33 & $\begin{array}{c}\text { Espécie descrita do Brasil: } \\
\text { São Paulo }\end{array}$ \\
\hline 27 & Neotropsylla guimaraesi & Polygenis guimaraesi & $\begin{array}{c}\text { Apenas } 2 \text { exemplares } \\
\text { conhecidos }\end{array}$ & ô 2,14 ㅇ 2,16 & $\begin{array}{l}\text { Espécie descrita do Brasil: } \\
\text { São Paulo }\end{array}$ \\
\hline
\end{tabular}


Tabela 1. Continuação...

\begin{tabular}{|c|c|c|c|c|c|}
\hline $\mathbf{N}^{\circ}$. & Espécies & $\begin{array}{c}\text { Vicissitudes } \\
\text { nomenclaturais }\end{array}$ & Distribuição dos sexos & $\begin{array}{c}\text { Dimensões médias } \\
(\mathbf{m m})\end{array}$ & Observações \\
\hline 28 & $\begin{array}{l}\text { Polygenis (Polygenis) } \\
\text { adelus }\end{array}$ & $\begin{array}{l}\text { Rhopalopsyllus adelus, } \\
\text { Polygenis versuta }\end{array}$ & Relação $+/ \widehat{\jmath}: 1,11$ & ภ $2,28+2,61$ & $\begin{array}{l}\text { Espécie descrita do Brasil: } \\
\text { São Paulo }\end{array}$ \\
\hline 29 & $\begin{array}{l}\text { Polygenis (Polygenis) } \\
\text { axius axius }\end{array}$ & $\begin{array}{l}\text { Rhopalopsyllus axius, } \\
\text { Polygenis gardellai }\end{array}$ & - & त 2,12 ㅇ 2,86 & - \\
\hline 30 & $\begin{array}{l}\text { Polygenis (Polygenis) a. } \\
\text { proximus }\end{array}$ & Polygenis axius proxima & Relação $+/ \delta: 0,25$ & 今 $2,20+2,75$ & $\begin{array}{c}\text { Espécie descrita do Brasil: } \\
\text { Mato Grosso }\end{array}$ \\
\hline 31 & $\begin{array}{l}\text { Polygenis (P.) o. } \\
\text { occidentalis }\end{array}$ & $\begin{array}{l}\text { Rothschildella occidentalis, } \\
\text { Rhopalosyllus plaumanni }\end{array}$ & Relação $+/ \delta: 2,60$ & त 2,18 ㅇ 2,64 & $\begin{array}{c}\text { Espécie descrita do Brasil: } \\
\text { São Paulo }\end{array}$ \\
\hline 32 & Polygenis (P.) rimatus & $\begin{array}{l}\text { Rhopalopsyllus bohlsi, } R \text {. } \\
\text { rimatus, Polygenis atra }\end{array}$ & Relação + O/ & त 1,90 ㅇ 2,22 & - \\
\hline 33 & $\begin{array}{l}\text { Polygenis }(P .) \text { roberti } \\
\text { roberti }\end{array}$ & $\begin{array}{c}\text { Pulex roberti, } \\
\text { Rhopalopsyllus roberti }\end{array}$ & Relação $+/ \delta: 1,20$ & 今 $2,53+2,74$ & $\begin{array}{l}\text { Espécie descrita do Brasil: } \\
\text { São Paulo }\end{array}$ \\
\hline 34 & Polygenis (P.) tripus & Rhopalopsyllus tripus & Relação $+/ \widehat{\jmath}: 1,51$ & त 2,08 ㅇ 2,26 & - \\
\hline 35 & $\begin{array}{l}\text { Polygenis (Neopolygenis) } \\
\text { atopus }\end{array}$ & $\begin{array}{l}\text { Rhopalopsyllus atopus, } R \text {. } \\
\text { agilis, } R \text {. truncatus }\end{array}$ & 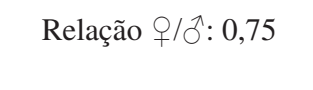 & $\lesssim 2,24$ ㅇ 2,55 & $\begin{array}{c}\text { Espécie descrita do Brasil: } \\
\text { Santa Catarina }\end{array}$ \\
\hline 36 & $\begin{array}{l}\text { Polygenis (Neopolygenis) } \\
\text { pradoi }\end{array}$ & Rhopalopsyllus pradoi & Relação + †/ठ: 1,93 & ठ̂ $2,20+2,41$ & $\begin{array}{c}\text { Espécie descrita do Brasil: } \\
\text { Santa Catarina }\end{array}$ \\
\hline 37 & Polygenis (N.) frustratus & Polygenis frustratus & Relação + †/ठ: 1,22 & ô 2,34 q 2,32 & $\begin{array}{c}\text { Espécie descrita do Brasil: } \\
\text { Santa Catarina }\end{array}$ \\
\hline 38 & Polygenis (N.) dentei & $\begin{array}{c}\text { Polygenis dentei, Polygenis } \\
\text { pygaerus dentei }\end{array}$ & Relação $+/$ ^: 0,75 & đ2,26 $+2,61$ & $\begin{array}{l}\text { Espécie descrita do Brasil: } \\
\text { São Paulo }\end{array}$ \\
\hline
\end{tabular}

\section{Lista das Espécies de Siphonaptera do Estado de São Paulo}

\section{Comentários sobre a lista, riqueza do Estado comparado com outras regiões.}

Conforme apresentado na lista e Tabela 2, a riqueza do estado totaliza 38 espécies e/ou subespécies presentemente assinaladas. Excetuando-se nove espécies cosmopolitas ou com distribuição geográfica estendendo-se por mais de uma região zoogeográfica, as 29 remanescentes $(76,3 \%)$ ocorrem exclusivamente na região Neotropical; quatro $(10,5 \%)$ são endêmicas em São Paulo, duas se distribuem pela região sudeste (ou sul) brasileira, cinco podem ser encontradas em todo o Brasil, 16 se estendem por toda a América do Sul e outras duas por toda a região Neotropical. A espécie com maior registro de localidades de ocorrência é $C$. felis felis, muito mais pela sua importância parasitológica e experimentos realizados para seu controle do que, propriamente, por pesquisa taxonômica. Com esta finalidade, Polygenis (Polygenis) roberti roberti (Rothschild, 1905), Polygenis (Polygenis) rimatus (Jordan, 1932) e Polygenis (N.) atopus têm sido as mais encontradas.

Em todo o Brasil, até o presente e excetuando-se S. pallidus, 62 espécies e/ou subespécies de pulgas foram assinaladas, incluídas em 19 gêneros e oito famílias, dos quais 14 gêneros $(73,7 \%)$ e todas as famílias ocorrem no estado de São Paulo. A sifonapterofauna de São Paulo representa 61,3\% (38/62) da brasileira, sendo a mais rica quando comparada com outros estados, seguida por Minas Gerais (54,8\%: 34/62), Rio de Janeiro (41,9\%: 26/62), Paraná (37,1\%: 23/62) e Santa Catarina (33,9\%: 21/62). A similaridade faunística entre São Paulo e seus estados limítrofes, baseada em espécies de pulgas e utilizando-se o coeficiente de Jaccard, representa $67,4 \%$ com Minas Gerais, 56,1\% (Rio de Janeiro), 45,2\% (Paraná) e 12,5\% (Mato Grosso do Sul).
Até agora, um total de 344 localidades e/ou municípios brasileiros proporcionaram registros para pulgas, ainda que de uma mesma localidade possam ter sido obtidos registros para mais de uma espécie de pulga. Assim, 173 registros foram já obtidos em diferentes localidades ou municípios do estado de São Paulo. Também considerando apenas o número de municípios onde pulgas já foram coletadas, o estado de São Paulo lidera, juntamente com Minas Gerais, no número de registros divulgados: 50 (14,5\%), seguido por Pernambuco (11,9\%), Paraná $(8,1 \%)$, Rio de Janeiro $(7,0 \%)$ e Bahia $(6,4 \%)$. Em parte, isto confirma a ideia de que

"a distribuição geográfica de um táxon (ou de uma doença) por localidades espelha, em grande parte dos casos, o número de pesquisadores (ou capturadores) que trabalham (ou se aglutinam) em dada região" (Linardi 1987a).

Aliás, é interessante salientar que qualquer animal, seja ele ectoparasito ou mamífero, não conhece os limites políticos entre localidades, municípios, estados ou países, estando a sua ocorrência na dependência de biomas ou domínios morfo-climáticos, alguns deles em continuidade. Consequentemente, ao se fazer estimativas sobre o número de espécies que devam ocorrer no estado de São Paulo, isto deve ser levado em consideração!

Em toda região Neotropical, excetuando-se a porção mexicana, ocorrem 52 gêneros e cerca de 280 espécies de sifonápteros. O número de espécies e/ou subespécies assinalado no Brasil é pequeno, quando comparado com outros países do Novo Mundo de menor extensão territorial e ocorrência de biomas: 37 no Panamá (Tipton \& Méndez 1966), 52 na Venezuela (Tipton \& Machado-Allison 1972), 44 na Colômbia (Méndez 1977), 134 no México (MoralesMuciño \& Llorente-Bousquets 1986), 108 na Argentina (Autino \& Lareschi 1998), 41 no Equador (Defense... 1998), 94 no Chile (Hastriter 2001) e 81 no Peru (Hastriter et al. 2002). Na Guiana 
Linardi, P.M

Tabela 2. Hospedeiros e distribuição geográfica das espécies de Siphonaptera do Estado de São Paulo, Brasil.

Table 2. Hosts and geographic distribution of the species of Siphonaptera from São Paulo State, Brazil.

\begin{tabular}{|c|c|c|c|c|c|}
\hline \multirow[b]{2}{*}{$\mathbf{N}^{\circ}$. } & \multirow[b]{2}{*}{ Espécies de Siphonaptera } & \multicolumn{2}{|c|}{ Hospedeiros primários } & \multicolumn{2}{|c|}{ Distribuição geográfica } \\
\hline & & Grupo taxonômico & Principais gêneros & $\begin{array}{c}\text { Regiões/Continentes/ } \\
\text { Países/Estados } \\
\end{array}$ & $\begin{array}{l}\text { No. Localidades } \\
\text { S. Paulo } \\
\end{array}$ \\
\hline 1 & Pulex irritans & $\begin{array}{l}\text { Carnivora, Primates, } \\
\text { Livre, Rodentia }\end{array}$ & Homo & Cosmopolita & 4 \\
\hline 2 & Xenopsylla cheopis & Rodentia: Muridae & Rattus, Mus & Cosmopolita & 4 \\
\hline 3 & Xenopsylla brasiliensis & Rodentia: Muridae & Rattus, Mus & $\begin{array}{c}\text { Etiópica, Oriental, Neotropical, } \\
\text { Paleártica }\end{array}$ & 2 \\
\hline 4 & Ctenocephalides canis & Carnivora & Canis & Cosmopolita & 2 \\
\hline 5 & Ctenocephalides felis felis & Carnivora & Canis, Felis & Cosmopolita & 15 \\
\hline 6 & Tunga penetrans & $\begin{array}{c}\text { Artiodactyla, Primates, } \\
\text { Rodentia }\end{array}$ & Sus, Homo & Neotropical, Etiópica & 6 \\
\hline 7 & Tunga caecata & Rodentia: Muridae & Rattus & Brasil & 3 \\
\hline 8 & Tunga travassosi & Pilosa & Dasypus & Endêmica em S. Paulo & 1 \\
\hline 9 & Tunga bondari & Pilosa & Tamandua & Brasil & 1 \\
\hline 10 & Tunga terasma & Cingulata & $\begin{array}{l}\text { Cabassous, } \\
\text { Euphractus }\end{array}$ & América do Sul & 1 \\
\hline 11 & Tunga trimamillata & Artiodactyla, Primates & $\begin{array}{l}\text { Bos, Capra, Homo, } \\
\text { Sus }\end{array}$ & América do Sul & 1 \\
\hline 12 & Hectopsylla psittaci & Aves & $\begin{array}{c}\text { Chalybea, Progne, } \\
\text { Turdus }\end{array}$ & Neotropical, Paleártica & 2 \\
\hline 13 & Hectopsylla pulex & Chiroptera & Eptesicus, Molossus & Neotropical & 3 \\
\hline 14 & Nosopsyllus (N.) fasciatus & Rodentia: Muridae & Rattus & Américas, Eurásia, Austrália & 1 \\
\hline 15 & Leptopsylla (L.) segnis & Rodentia: Muridae & Mus & Cosmopolita & 2 \\
\hline 16 & Hormopsylla fosteri & Chiroptera & $\begin{array}{l}\text { Molossus, } \\
\text { Phylostoma }\end{array}$ & América do Sul & 1 \\
\hline 17 & $\begin{array}{l}\text { Craneopsylla minerva } \\
\text { minerva }\end{array}$ & Rodentia: Cricetidae & Akodon, Oryzomys & Neotropical & 9 \\
\hline 18 & $\begin{array}{l}\text { Adoratopsylla (A.) a. } \\
\text { antiquorum }\end{array}$ & Rodentia: Cricetidae & $\begin{array}{l}\text { Oryzomys, } \\
\text { Thomasomys }\end{array}$ & América do Sul & 8 \\
\hline 19 & $\begin{array}{l}\text { Adoratopsylla (A.) a. } \\
\text { ronnai }\end{array}$ & Didelphimorphia & $\begin{array}{l}\text { Didelphis, } \\
\text { Philander }\end{array}$ & Sul e Sudeste do Brasil & 1 \\
\hline 20 & $\begin{array}{l}\text { A. (Tritopsylla) } i . \\
\text { intermedia }\end{array}$ & $\begin{array}{l}\text { Didelphimorphia, } \\
\text { Rodentia }\end{array}$ & $\begin{array}{l}\text { Didelphis, } \\
\text { Marmosa, } \\
\text { Oryzomys }\end{array}$ & América do Sul & 8 \\
\hline 21 & Rhopalopsyllus lutzi lutzi & $\begin{array}{c}\text { Pilosa, } \\
\text { Didelphimorphia, } \\
\text { Livres }\end{array}$ & Dasypus, Didelphis & América do Sul & 8 \\
\hline 22 & $\begin{array}{l}\text { Rhopalopsyllus lugubris } \\
\text { lugubris }\end{array}$ & $\begin{array}{c}\text { Rodentia: } \\
\text { Hystricomorpha }\end{array}$ & Cuniculus & América do Sul & 4 \\
\hline 23 & $\begin{array}{l}\text { Rhopalopsyllus australis } \\
\text { tamoyus }\end{array}$ & $\begin{array}{c}\text { Rodentia: } \\
\text { Hystricomorpha }\end{array}$ & Dasyprocta & América do Sul & 1 \\
\hline 24 & R. australis tupiniquinus & Carnivora & Eira & Endêmica em S. Paulo & 1 \\
\hline 25 & Hechtiella lakoi & $\begin{array}{l}\text { Rodentia: } \\
\text { Hystricomorpha }\end{array}$ & Trinomys & Brasil & 1 \\
\hline 26 & Hechtiella lopesi & $\begin{array}{c}\text { Rodentia: } \\
\text { Hystricomorpha }\end{array}$ & Trinomys & Endêmica em S. Paulo & 2 \\
\hline 27 & Neotropsylla guimaraesi & Rodentia: Cricetidae & Calomys & Endêmica em S. Paulo & 1 \\
\hline 28 & $\begin{array}{l}\text { Polygenis (Polygenis) } \\
\text { adelus }\end{array}$ & Rodentia & $\begin{array}{l}\text { Trinomys, } \\
\text { Oryzomys }\end{array}$ & Brasil & 1 \\
\hline 29 & $\begin{array}{l}\text { Polygenis (Polygenis) axius } \\
\text { axius }\end{array}$ & Rodentia: Cricetidae & Oxymycterus & América do Sul & 2 \\
\hline
\end{tabular}


Tabela 2. Continuação...

\begin{tabular}{|c|c|c|c|c|c|}
\hline \multirow[b]{2}{*}{$\mathbf{N}^{\circ}$} & \multirow[b]{2}{*}{ Espécies de Siphonaptera } & \multicolumn{2}{|c|}{ Hospedeiros primários } & \multicolumn{2}{|c|}{ Distribuição geográfica } \\
\hline & & Grupo taxonômico & Principais gêneros & $\begin{array}{c}\text { Regiões/Continentes/ } \\
\text { Países/Estados }\end{array}$ & $\begin{array}{l}\text { No. Localidades } \\
\text { S. Paulo } \\
\end{array}$ \\
\hline 30 & $\begin{array}{l}\text { Polygenis (Polygenis) a. } \\
\text { proximus }\end{array}$ & Rodentia: Cricetidae & Akodon & Brasil & 1 \\
\hline 31 & $\begin{array}{l}\text { Polygenis }(P .) \text { o. } \\
\text { occidentalis }\end{array}$ & Rodentia & Sciurus & América do Sul & 6 \\
\hline 32 & Polygenis (P.) rimatus & Rodentia: Cricetidae & Akodon, Oryzomys & América do Sul & 10 \\
\hline 33 & $\begin{array}{l}\text { Polygenis }(P .) \text { roberti } \\
\text { roberti }\end{array}$ & Rodentia: Cricetidae & Oryzomys & América do Sul & 15 \\
\hline 34 & Polygenis (P.) tripus & Rodentia: Cricetidae & Akodon & América do Sul & 9 \\
\hline 35 & $\begin{array}{l}\text { Polygenis (Neopolygenis) } \\
\text { atopus }\end{array}$ & Rodentia: Cricetidae & Oryzomys & América do Sul & 13 \\
\hline 36 & $\begin{array}{l}\text { Polygenis (Neopolygenis) } \\
\text { pradoi }\end{array}$ & Rodentia: Cricetidae & Akodon & América do Sul & 8 \\
\hline 37 & Polygenis (N.) frustratus & Rodentia: Cricetidae & Akodon & América do Sul & 2 \\
\hline 38 & Polygenis (N.) dentei & Rodentia: Cricetidae & $\begin{array}{l}\text { Akodon, } \\
\text { Thomasomys }\end{array}$ & Sudeste do Brasil & 2 \\
\hline
\end{tabular}

Francesa, Beaucournu et al. (1998) estimam a sifonapterofauna em, aproximadamente, 15 espécies.

\section{Principais avanços relacionados ao Programa BIOTA/FAPESP}

Ainda que o número de espécies de pulgas no estado de São Paulo tenha aumentado de 36 para 38 nos últimos 11 anos, com a inclusão de Polygenis (Polygenis) adelus (Jordan \& Rothschild, 1923) e . trimamillata, os seguintes avanços foram obtidos em relação à sifonapterofauna:

- Descrição da respectiva fêmea de Polygenis (Neopolygenis) dentei Guimarães, 1947 do Parque Nacional do Caparaó/MG, o que veio confirmar a sua identidade específica, já que anteriormente era considerada subespécie e conhecida apenas por três exemplares machos, todos eles capturados na Estação Biológica de Boracéia, Salesópolis/SP;

- Novos registros de hospedeiros (27 diferentes espécies de roedores silvestres ou marsupiais) para 17 diferentes espécies de pulgas;

- Novos registros de ocorrência de espécies de pulgas para 17 municípios, especialmente em áreas de conservação, como a Estação Ecológica Juréia-Itatins, o Parque Nacional da Serra da Bocaina, o Parque Estadual de Cantareira, ou mesmo a Floresta Atlântica da Serra da Fartura;

- Estudos ecológicos e parasitológicos complementares aos taxonômicos, no que diz respeito às interações pulgas/hospedeiros: prevalência, abundância, intensidade de infestação e especificidade parasitária nas regiões em foco;

- Constatação de infecção natural por Rickettsia felis em uma dada espécie de pulga: $C$. felis felis e a possibilidade desta mesma espécie poder atuar como vetor mecânico na transmissão do calazar canino.

Cumpre salientar que em relação aos seis artigos publicados nos últimos 10 anos versando sobre pulgas e outros ectoparasitos associados, ou mesmo objetivando as pulgas como vetoras de agentes infecciosos, cinco foram desenvolvidos com recursos da FAPESP, dois destes como produto de teses de doutorado.

\section{Principais grupos de pesquisa}

Após o falecimento de Lindolpho Rocha Guimarães em 1998, não há qualquer grupo de pesquisa trabalhando exclusivamente com taxonomia de sifonápteros no estado de São Paulo. As poucas contribuições posteriores dizem respeito a ectoparasitos em geral, em que alguns sifonápteros têm sido colecionados e identificados juntamente com ácaros, ou enfocando as pulgas como hospedeiras de agentes infecciosos.

\section{Principais acervos}

O principal acervo de sifonápteros no estado de São Paulo é, sem dúvida, a coleção de ectoparasitos do Museu de Zoologia da Universidade de São Paulo, organizada por L.R. Guimarães ao longo de quase 70 anos, inicialmente integrando o Departamento de Zoologia da Secretaria de Agricultura do Estado de São Paulo. Ela possui excelente infra-estrutura e, à exceção de duas espécies de pulgas, contém outras 36 que ocorrem em São Paulo, além de praticamente todos os representantes da sifonapterofauna brasileira. Nas fichas e registros relativos às espécies de pulgas depositadas no Museu de Zoologia há informações sobre material também estudado ou armazenado da coleção de Flávio da Fonseca, do Instituto Butantan/SP. Ao menos, 20 diferentes coletores colaboraram ao longo do tempo na ampliação do acervo do MZUSP.

\section{Principais lacunas do conhecimento}

Uma das principais dificuldades para o estudo dos Siphonaptera está relacionada à obtenção de material para estudo, já que ela é indireta e depende da captura dos respectivos hospedeiros. Cada grupo de hospedeiro merece tratamento especial para a sua captura, a considerar os hábitos, hábitats, nichos e os diferentes sítios de parasitismo pelo corpo. A maioria dos hospedeiros deve ser capturada utilizando-se de armadilhamentos. As armadilhas devem estar regularmente espaçadas sobre o terreno e em bom número, na região investigada. A eficiência da captura será aumentada caso as trilhas e o interior das armadilhas sejam cevadas, anteriormente à sua armação. Uma vez capturados, nem sempre os hospedeiros se apresentarão parasitados, desde que a infestação pode estar 
relacionada com fatores climáticos e/ou ecológicos (competição, predação entre ectoparasitos). Ao contrário de outros ectoparasitos como piolhos e ácaros, as pulgas fogem do corpo dos hospedeiros tão logo sejam capturados, razão pela qual a despulização deve ser processada imediatamente após a captura ou aprisionamento dos respectivos hospedeiros.

Entre as espécies já assinaladas no estado, apenas cinco $(13,1 \%)$ foram descritas nos últimos 60 anos, com a maioria tendo sido conhecida entre 1901 e 1950: 65,8\% (25) e a última, Hechtiella lopesi Guimarães \& Linardi, 1993, há quase duas décadas. É importante assinalar que o crescimento da sifonapterofauna deve ser creditado mais à sucessiva adição de novos registros geográficos, antes que à descrição de novos táxons.

Das 62 espécies e subespécies aqui existentes, apenas 16 (25,8\%) foram descritas por autores nacionais. Isto talvez em decorrência do fato de que, até a metade deste século, vários grupos internacionais patrocinavam expedições científicas ao Brasil, para cá enviando capturadores profissionais para extraírem nossas riquezas naturais. Consequentemente, não apenas pulgas, mas outras espécies de insetos foram assim descritas, em virtude desta ação predatória contra nosso patrimônio zoológico. Do ponto de vista científico, seria assaz importante que expedições visando o estudo de nossas fauna e flora fossem lideradas por pesquisadores pátrios!

Três espécies, respectivamente, Tunga caecata (Enderlein, 1901), Tunga travassosi Pinto \& Dreyfus, 1927 e Tunga bondari Wagner, 1932, as duas primeiras descritas de São Paulo, são conhecidas apenas por exemplares fêmeas. Em outra, Hectopsylla pulex (Haller, 1880), os machos são observados apenas no guano dos respectivos hospedeiros. Para duas outras espécies, Rhopalopsyllus australis tupinus Jordan \& Rothschild, 1923 e Neotropsylla guimaraesi (Linardi, 1978), até o presente, menos de três exemplares foram capturados.

As 10 espécies descritas de São Paulo foram provenientes dos seguintes municípios: T. caecata (Piracicaba), T. travassosi (Sorocaba), Rhopalopsyllus lutzi lutzi (Baker, 1904) (São Paulo), Rhopalopsyllus australis tupiniquinus Guimarães, 1940 (Franca), H. lopesi (Salesópolis), N. guimaraesi (Itapetininga), P. (P.) adelus (Porto Martins), Polygenis (Polygenis) occidentalis occidentalis (Cunha, 1914) (São Paulo), P. (P.) R Roberti (São Paulo) e $P$. (N.) dentei (Salesópolis). Três destas, H. lopesi, R. a. tupiniquinus e $N$. guimaraesi, só são conhecidas das respectivas localidades de onde foram descritas.

\section{Perspectivas de pesquisa em Siphonaptera para os próximos 10 anos}

Do ponto de vista taxonômico, os futuros estudos deveriam enfocar, essencialmente, as espécies das famílias Rhopalopsyllidae, Tungidae, Stephanocircidae, Ischnopsyllidae e da tribo Tritopsyllini (Ctenophthalmidae), típicas da região Neotropical. Entre as 31 espécies e/ou subespécies descritas do Brasil, 17 são endêmicas (54,8\%), algumas destas com um único registro de localidade, município ou estado, sendo 12 na região sudeste e quatro no estado de São Paulo.

Os futuros objetivos deveriam ser direcionados às regiões pouco exploradas, em especial aquelas onde alguns táxons foram descritos, estudando-se o ectoparasito como um todo. Seria de interesse salientar que a Estação Ecológica de Boracéia, em Salesópolis, tendo em vista o achado de duas novas espécies para a Ciência, bem como o registro de vários outros táxons, alguns exibindo características intermediárias entre duas espécies de um determinado complexo, denominado "pradoi" (Linardi 1979), constitui um ambiente profícuo para maiores investigações taxonômicas.

No que concerne aos registros, seria essencial que as localidades fossem referenciadas por suas coordenadas geográficas, tendo em vista a multiplicidade de localidades brasileiras com um mesmo nome, mudanças nomenclaturais de uma mesma localidade e possíveis transliterações erradas.

A identificação dos hospedeiros deve ser feita criteriosamente, por especialistas, uma vez que as vicissitudes nomenclaturais são muito comuns em certos grupos de mamíferos, especialmente roedores e marsupiais. Uma vez que as várias listagens de hospedeiros contidas em quase todas as publicações taxonômicas não separam os verdadeiros hospedeiros de outros supostamente tidos como secundários ou acidentais, as pesquisas neste campo deveriam ser canalizadas no sentido da exata determinação dos respectivos conteúdos de precipitina. Da mesma forma, dados sobre prevalência, abundância ou intensidade de infestação deveriam acompanhar as informações taxonômicas, já que métodos estatísticos também podem, indiretamente, separar as categorias de hospedeiros. Ressalte-se que o reconhecimento de hospedeiros verdadeiros e a subsequente atribuição de categorias, como hospedeiros exclusivos, primitivos e/ou primários é passo fundamental para estudos de co-evolução que poderão refletir histórias filogenéticas comuns.

Os estudos de campo devem se estender aos ninhos dos hospedeiros, visando à obtenção de formas imaturas. Não mais que 60 larvas de pulgas são conhecidas em todo o mundo. E quase nada se conhece sobre a morfologia das larvas de pulgas neotropicais. Isto poderia oferecer subsídios à sistemática, tendo em vista o relevante papel epidemiológico que algumas dessas espécies desempenham. Alguns projetos sobre o desenvolvimento de ciclos biológicos poderiam ser tentados, destacando-se a influência de certos fatores nutricionais e ambientais para a sua complementação.

Por outro lado, sendo os índices pulidicianos um referencial para a vigilância à peste, os dados relativos às pulgas encontradas fora dos hospedeiros seriam de especial valor. Pulgas adultas, especialmente as de ocorrência neotropical, também poderiam ser examinadas em relação ao seu papel na transmissão de agentes infecciosos.

Recentemente, Cardoso \& Linardi (2006) descreverem algumas ultra-estruturas de Polygenis (Polygenis) tripus (Jordan, 1933) por meio de microscopia eletrônica de varredura, constituindo-se no primeiro registro para pulgas Rhopalopsyllidae, bem como na terceira caracterização ultra-estrutural de espécies neotropicais de pulgas, após $T$. penetrans e $T$. trimamillata.

Finalmente, espera-se para os próximos 10 anos, uma maior aplicação da biologia molecular na resolução de questões taxonômicas, ou mesmo, complementando estudos clássicos com base em morfologia.

\section{Referências Bibliográficas}

ALMEIDA, F.A. \& CROCE, J. 1990. Hipersensibilidade em pacientes com prurigo de Hebra causado por picada de pulga. Med. Cut. Ibero Lat. Am. 18:132-7.

AUTINO, A.G. \& LARESCHI, M. 1998. Siphonaptera. In Biodiversidad de artrópodos argentinos. Una perspectiva biotaxonómica. (J.J. Morrone \& S. Coscarón, ed.). Ed. Sur, La Plata, p.279-290.

AVELAR, D.M. 2010. Sistemática e análise cladística das espécies neotropicais do gênero Tunga Jarocki, 1838 (Siphonaptera: Tungidae). Tese de doutorado, Universidade Federal de Minas Gerais.

BARNES, A.M. \& RADOVSKY, F.J. 1969. A new Tunga (Siphonaptera) from the Neartic region with description of all stages. J. Med. Entomol. 6(1):19-36. PMid:5775471.

BARROS-BATTESTI, D.M., LINARDI, P.M. \& ARZUA, M. 1997. Levantamento das espécies de pulgas coletadas em pequenos mamíferos capturados em área de mata atlântica no município de Itapevi, SP. In $15^{\circ}$ Congresso Brasileiro de Parasitologia, Salvador, p.234. 
BEAUCOURNU, J.C., REYNES, J.M. \& VIÉ, J.C. 1998. Fleas in French Guiana (Insecta: Siphonaptera). J. Med. Entomol. 35(1):3-10. PMid:9542339.

BOSSI, D.E.P. 1996. Ectoparasitismo de pequenos mamíferos da Estação Ecológica de Juréia-Itatins, Iguape (SP). Tese de mestrado, Universidade Estadual de Campinas.

BOSSI, D.E.P. 2003. Associações entre artrópodes e pequenos mamíferos silvestres de três áreas serranas do sudeste brasileiro. Tese de doutorado, Universidade Estadual de Campinas.

BOSSI, D.E.P., LINHARES, A.X. \& BERGALLO, H.G. 2002. Parasitic arthropods of some wild rodents from Juréia-Itatins Ecological Station, State of São Paulo, Brazil. Mem. Inst. Oswaldo Cruz 97(7):959-963. PMid:12471421.

CARDOSO, V.A. \& LINARDI, P.M. 2006. Scanning electron microscopy studies of sensilla and other structures of the head of Polygenis (Polygenis) tripus (Siphonaptera: Rhopalopsyllidae). Micron 37:557-565. http:// dx.doi.org/10.1016/j.micron.2005.12.001

COUTINHO, M.T.Z. \& LINARDI, P.M. 2007. Can fleas from dogs infected with canine visceral leishmaniasis transfer the infection to other mammals? Vet. Parasitol. 147(3-4):320-5. http://dx.doi.org/10.1016/j. vetpar.2007.04.008

COUTINHO, M.T.Z. 2003. Investigação da capacidade vetorial de sifonápteros e ixodídeos na leishmaniose visceral canina. Tese de doutorado, Universidade Federal de Minas Gerais.

CUNHA, R.A. 1914. Contribuição para o estudo dos sifonapteros do Brasil. Instituto Oswaldo Cruz, Rodrigues e C., Rio de Janeiro.

DEFENSE PEST MANAGEMENT INFORMATION ANALYSIS CENTER - DPMIAC. 1998. Disease vector. Ecology profile Ecuador. Walter Reed Army Medical Center, Washington. http.//www.afpmb.org/pubs/dveps/ Ecuador.pdf (último acesso em 28/06/2010).

FERREIRA, M.G.P.A., FATTORI, K.R., SOUZA, F. \& LIMA, V.M.F. 2009 Potential role for dog fleas in the cycle of Leishmania spp. Vet. Parasitol. 165(1-2):150-154. http://dx.doi.org/10.1016/j.vetpar.2009.06.026

FONSECA, F. \& PRADO, A. 1932. Algumas verificações parasitológicas em ratos de São Paulo. Rev. Med. Cirurg. Brasil. 40:65-70.

GUIMARÃES, L.R. 1936a. Notas sobre a destruição de pulgas por meio de inseticidas. Arch. Hyg. Saúde Publ. S. Paulo 1:55-60.

GUIMARÃES, L.R. 1936b. Notas sobre siphonapteros com a descrição de uma nova espécie. Arch. Hyg. Saúde Publ. S. Paulo 1(2):141-143.

GUIMARÃES, L.R. 1938. Sobre a incidência de pulgas em ratos na cidade de Santos. Ann. Paul. Med. Cir. 36(3):283-289.

GUIMARÃES, L.R. 1940. Notas sobre Siphonaptera e redescrição de Polygenis occidentalis (Almeida Cunha, 1914). Arq. Zool. Est. S. Paulo 2(6):215-250.

GUIMARÃES, L.R. 1942. Sobre algumas espécies de pulgas brasileiras. Pap. Av. Dep. Zool. S. Paulo 2(15):197-203.

GUIMARÃES, L.R. 1944. Ectoparasitos de aves e mamíferos colecionados em Monte Alegre. Pap. Av. Dep. Zool. S. Paulo 6(2):15-20.

GUIMARÃES, L.R. 1945. Alguns aspectos bionômicos de Leptopsylla segnis (Schönh) (Suctoria). Arq. Zool. Est. S. Paulo 4(7):233-240.

GUIMARÃES, L.R. 1947. Duas novas espécies de Polygenis Jordan, 1939 (Pulicidae-Suctoria). Pap. Av. Dep. Zool. S. Paulo 8(15):189-195.

GUIMARÃES, L.R. 1948. Sôbre algumas espécies do gênero Polygenis Jordan, 1939 (Pulicidae-Suctoria). Arq. Zool. Est. S. Paulo 5(8):539-552.

GUIMARÃES, L.R. 1954. Notas sobre algumas espécies de Hystrichopsyllidae (Siphonaptera) da América do Sul. Pap. Av. Dep. Zool. Est. S. Paulo 11(26):509-515.

GUIMARÃES, L.R. 1956. Nova espécie de pulga do gênero Polygenis Jordan, 1939 (Rhopalopsyllidae). Rev. Bras. Malariol. D. Trop. 3(1):172-174.
GUIMARÃES, L.R. \& LINARDI, P.M. 1993. Hechtiella lopesi sp. n. from São Paulo State, Brazil (Siphonaptera: Rhopalopsyllidae). Mem. Inst. Oswaldo Cruz 88(4):547-550. http://dx.doi.org/10.1590/S007402761993000400008

HASTRITER, M.W. 2001. Fleas (Siphonaptera: Ctenophthalmidae and Rhopalopsyllidae) from Argentina and Chile with two new species from the rock rat, Aconaemys fuscus, in Chile. Ann. Carn. Mus. 70(2):169-178.

HASTRITER, M.W., ZYZAK, M.D., SOTO, R., FERNANDEZ, R., SOLORZANO, N. \& WHITING, M. 2002. Fleas (Siphonaptera) from Ancash Department Peru with the description of a new species, Ectinorus alejoi (Rhopalopsyllidae), and the description of the male of Plocopsylla pallas (Rothschild, 1914) (Stephanocircidae). Ann. Carn. Mus. 71(2):87-106

HICKS, E.P. 1930. The early stages of the jigger, Tunga penetrans. Ann. Trop. Med. Parasit. 24:576-586.

HOPKINS, G.H.E. \& ROTHSCHILD, M. 1953. An illustrated catalogue of the Rothschild Collection of fleas (Siphonaptera) in the British Museum (Natural History). British Museum (Natural History), London. v.1.

HOPKINS, G.H.E. \& ROTHSCHILD, M. 1956. An illustrated catalogue of the Rothschild Collection of fleas (Siphonaptera) in the British Museum (Natural History). British Museum (Natural History), London. v.2.

HOPKINS, G.H.E. \& ROTHSCHILD, M. 1962. An illustrated catalogue of the Rothschild Collection of fleas (Siphonaptera) in the British Museum (Natural History). British Museum (Natural History), London. v.3.

HOPKINS, G.H.E. \& ROTHSCHILD, M. 1966. An illustrated catalogue of the Rothschild Collection of fleas (Siphonaptera) in the British Museum (Natural History). British Museum (Natural History), London. v.4.

HOPKINS, G.H.E. \& ROTHSCHILD, M. 1971 An illustrated catalogue of the Rothschild Collection of fleas (Siphonaptera) in the British Museum (Natural History). British Museum (Natural History), London. v.5.

HORTA, M.C. 2006. Estudo epidemiológico de Rickettsia felis em áreas endêmicas e não endêmicas para febre maculosa no Estado de São Paulo. Tese de doutorado, Universidade de São Paulo.

HORTA, M.C., LABRUNA, M.B., PINTER, A., LINARDi, P.M. \& SCHUMAKER, T.T.S. 2007. Rickettsia infection in five areas of the state of São Paulo, Brazil. Mem. Inst. Oswaldo Cruz 102(7):793-801. http:// dx.doi.org/10.1590/S0074-02762007000700003

JOHNSON, P.T. 1957. A classification of Siphonaptera of South America with descriptions of new species. Mem. Ent. Soc. Wash. 5:1-298.

KRASNOV, B. 2008. Functional and evolutionary ecology of fleas. A model for ecological parasitology. Cambridge University Press, New York. http:// dx.doi.org/10.1017/CBO9780511542688

LEWIS, R.E. 1998. Resumé of Siphonaptera (Insecta) of the world. J. Med. Entomol. 35(4):377-389.

LIMA, A.C. \& HATHAWAY, C.R. 1946. Pulgas, bibliografia, catálogo e hospedadores. Instituto Oswaldo Cruz, Rio de Janeiro.

LIMA, A.C. 1940. Nota sobre as especies de Tunga (Siphonaptera: Tungidae). Acta Medica 5:300-302.

LIMA, A.C. 1943. Suctoria. In Insetos do Brasil. Escola Nacional de Agronomia, Rio de Janeiro, p.17-71.

LINARDI, P.M. 1974. Sifonápteros de roedores dos municípios de Salesópolis e Itapetininga, SP. Tese de mestrado, Universidade Federal de Minas Gerais.

LINARDI, P.M. 1977. Relações pulgas/roedores observadas nos municípios de Salesópolis e Itapetininga, SP. Bol. Mus. Hist. Nat. UFMG Zool. 23:1-25.

LINARDI, P.M. 1978. Polygenis guimaraesi sp. n. from São Paulo, Brazil (Siphonaptera: Rhopalopsyllidae). Rev. Bras. Biol. 38(2):469-473.

LINARDI, P.M. 1979. Sobre algumas espécies de Rhopalopsyllidae (Siphonaptera) sulamericanas integrantes do "complexo pradoi". Revta. Bras. Ent. 23(2):99-106. 
LINARDI, P.M. 1981a. Utilização de algumas estruturas na caracterização de espécies da ordem Siphonaptera. I. A fratura da mesocoxa na separação de espécies de Polygenis Jordan, 1939. Rev. Bras. Ent. 25(1):27-29.

LINARDI, P.M. 1981b. Polygenis Jordan, 1939 (Siphonaptera: Rhopalopsyllidae). Estudos morfológicos, zoogeográficos, fenéticos, filogenéticos e relação hospedeiro/parasite. Tese de doutorado, Universidade Federal de Minas Gerais.

LINARDI, P.M. 1984a. Utilização de algumas estruturas na caracterização de espécies da ordem Siphonaptera. II. A quetotaxia da margem posterior da tíbia posterior na separação de espécies de Polygenis Jordan, 1939. Rev. Bras. Ent. 28(1):115-119.

LINARDI, P.M. 1984b. Utilização de algumas estruturas na caracterização de espécies da ordem Siphonaptera. III. A variabilidade do braço ventral do esternito IX em Poiygenis rimatus e suas inplicações taxonômicas. Rev. Bras. Ent. 28(3):261-262.

LINARDI, P.M. 1984c. Relações taxonômicas e filogenéticas entre os gêneros de sifonápteros ropalopsilinos obtidas das relações hospedeiro/parasito. Rev. Bras. Biol. 44(3):329-334.

LINARDI, P.M. 1985a. Dados complementares sobre hospedeiros de sifonápteros ropalopsilinos. Rev. Bras. Biol.45(1-2):73-78.

LINARDI, P.M. 1985b. Dados complementares sobre hospedeiros de sifonápteros ropalopsilinos. Rev. Bras. Biol.45(1-2):73-78.

LINARDI, P.M. 1987a. Distribuição geográfica dos sifonápteros ropalopsilinos. Rev. Bras. Biol. 47(3):385-396.

LINARDI, P.M. 1987b. Subsídios taxonômicos e filogenéticos obtidos por comparações faunísticas da distribuição geográfica dos sifonápteros ropalopsilinos. Rev. Bras. Biol. 47(3):397-407.

LINARDI, P.M. 1999. Siphonaptera. In Biodiversidade do Estado de São Paulo, Brasil, 5. Invertebrados terrestres (C.R.F. Brandão \& E.M. Cancello, ed.). Fapesp, São Paulo, p.265-275. PMid:8433323.

LINARDI, P.M. \& GUIMARÃES, L.R. 1993. Systematic review of genera and subgenera of Rhopalopsyllinae (Siphonaptera: Rhopalopsyllidae) by phenetic and cladistic methods. J. Med. Entomol. 30(1):161-170.

LINARDI, P.M. \& GUIMARÃES, L.R. 2000a. Sifonápteros do Brasil. Museu de Zoologia da USP; Fapesp, São Paulo.

LINARDI, P.M. \& GUIMARÃES, L.R. 2000b. Sifonápteros do Brasil. Museu de Zoologia da USP; Fapesp, São Paulo.

LINARDI, P.M. \& LARESCHI, M. 2003. Description of the female sex of Polygenis (Neopolygenis) dentei Guimarães, 1947 (Siphonaptera: Rhopalopsyllidae). Entom. Vect. 10(3):401-406.

LINARDI, P.M. \& NAGEM, R.L. 1972. Observações sobre o ciclo evolutivo de Ctenocephalides felis (Bouché, 1835) (Siphonaptera, Pulicidae) e sua sobrevida fora do hospedeiro. Bol. Mus. Hist. Nat. UFMG Zool. 13:1-22.

LUTZ, A.S.A. 1903. Nota preliminar sobre os insetos sugadores de sangue observados nos estados de São Paulo e Rio de Janeiro. Brasil-Méd. 17(29):281-282.

MACHADO-ALLISON, C.E. 1962. Notas sobre Rhopalopsyllidae. I. Sobre algumas formas de Polygenis Jordan (Siphonaptera, Rhopalopsylloidea). Pap. Av. Dep. Zool. S. Paulo 15(3):23-27.

MARSHALL, A.G. 1981. The ecology of ectoparasitic insects. Academic Press, London.

MASCARENHAS, R.C.S. 2002. A co-evolução de Tunginae (Siphonaptera Pulicidae) e os Edentata (Mammalia). Tese de doutorado, Universidade Estadual Paulista Júlio de Mesquita Filho.

MEDVEDEV, S.G. 1994. Morphological basis of classification of the order Siphonaptera. Entomol. Rev. 73:22-43.

MEDVEDEV, S.G. 2002. Specific features of the distribution and host associations of fleas (Siphonaptera). Entomol. Rev. 82:1165-1177.

MEIRA, J.A. 1931. Alguns dados estatísticos sobre as pulgas de ratos na cidade de São Paulo. Ann. Fac. Med. S. Paulo 6:73-77.
MEIRA, J.A. 1932. Alguns dados estatísticos sobre as pulgas de ratos na cidade de São Paulo (2a . Nota). Brasi-Méd. 46(19):429-432.

MEIRA, J.A. 1934. Contribuição parasitológica para a epidemiologia da peste bubônica na cidade de São Paulo. Sobre as pulgas de rato da mesma cidade. Ann. Paul. Med. Cir. 28(2):143-193.

MÉNDEZ, E. 1977. Mammalian-siphonapteran associations, the environment, and biogeography of mammals of Southwestern Colombia. Quaest. Entomol. 13:91-182.

MORAES, L.B., BOSSI, D.E.P. \& LINHARES, A.X. 2003. Siphonaptera parasites of wild rodents and marsupials trapped in three mountain ranges of the Atlantic Forest in southeastern Brazil. Mem. Inst. Oswaldo Cruz 98(8):1071-1076. http://dx.doi.org/10.1590/S0074-02762003000800017

MORALES-MUCIÑO, J.C. \& LLORENTE-BOUSQUETS, J. 1986. Estado actual del conocimento de los Siphonaptera de México. Ann. Inst. Biol. Univ. Nac. Auton. Mex. Ser. Zool. 56(2):497-554.

NAGEM, R.L. 1977. Sifonápteros da Coleção UFMG: sistemática, relações estruturais, adaptativas e entre hospedeiro/parasito. Tese de mestrado, Universidade Federal de Minas Gerais.

NIERI-BASTOS, F.A., BARROS-BATTESTI, D.M., BEGALE, F.F., LINARDI, P.M., MARCILI, A. \& FAVORITO, S.E. 2003. Lista preliminar da ectoparasitofauna de pequenos mamíferos terrestres de Biriba-Mirim e Paraitinga, São Paulo, Brasil. In $28^{\circ}$ Congresso Brasileiro de Parasitologia. Rio de Janeiro, p.87.

NIERI-BASTOS, F.A., BARROS-BATTESTI, D.M., LINARDI, P.M., AMAKU, M., MARCILI, A., FAVORITO, S.E. \& PINTO-DA-ROCHA, 2004a. Ectoparasites of wild rodents from Parque Estadual da Cantareira (Pedra Grande Nuclei), São Paulo, Bras. Rev. Brasil. Parasit. Vet. 13(1):29-35.

NIERI-BASTOS, F.A., ARZUA, M., BEGALE, F.F., GIACOMIN, F.G. \& BARROS-BATTESTI, D.M. 2004b. Siphonaptera de pequenos mamíferos silvestres (Rodentia e Didelphimorphia) do município de Itapevi, São Paulo, Brasil. In XII Congresso Brasileiro de Parasitologia Veterinária e I Simpósio Latino-Americano de Rickettsioses. Ouro Preto, p.333.

PACHECO, R.S., MARZOCHI, M.C.A., PIRES, M.Q., BRITO, C.M.M., MADEIRA, M.F. \& BARBOSA-SANTOS, E.G.O. 1998. Parasite genotypically retated to a monoxenous tripanosomatid of dog's flea causing opportunistic infection in an HIV positive patient. Mem Inst Oswaldo Cruz 93:531-7. http://dx.doi.org/10.1590/S007402761998000400021

PAMPIGLIONE, S., TRENTINI, M., FIORAVANTI, M.L., ONORE, G. \& RIVASI, F. 2002. A new species of Tunga (Insecta, Siphonaptera) in Ecuador. Parassitologia 44(supl.1):127.

PESSOA, S.B. \& HORTA, C.L. 1933. Nota sobre a evolução de algumas espécies de pulgas em São Paulo. Ann. Paul. Med. Cir. 25:3-4.

PINTO, C. \& DREYFUS, A. 1927. Tunga travassosi n. sp., parasita de Tatusia novemcinctus do Brasil. Bol. Biol. 9:174-178.

PINTO, C. 1925. Estudo sobre siphonapteros ou pulgas. Stenopsylla cunhai n. sp. Bol. Inst. Bras. Sci. 1:3-7.

PINTO, C. 1930. Arthrópodes parasitos e transmissores de doenças. Pimenta de Mello \& C., Rio de Janeiro.

PINTO, C. 1931. Características morfológicas da larva de Ctenocephalides felis (Bouché, 1835)(Siphonaptera, Pulicidae). Bol. Biol. 18:28-34.

PRADO, A. 1933. Uma nova pulga do gambá (Siphonaptera, Pulicidae). Rev. Bras. Ent. Rio de Janeiro 3(3):322-325.

ROTHSCHILD, M., SCHLEIN, Y., PARKER, K., NEVILLE, C. \& STERNBERG, S. 1973. The flying leap of the flea. Sci. Amer. 229:92-100. http://dx.doi.org/10.1038/scientificamerican1173-92 
SANT'ANNA, W.B., MOREIRA, G.M., MACHADO, P.F.O., TEIXEIRA, R.H.F., CARVALHO, R.W. \& SERRA-FREIRE, N.M. 2004. Ctenocephalides felis felis (Curtis, 1826) em Myrmecophaga trydactila e Didelphis marsupialis mantidos em cativeiro no zoológico de São Paulo, Brasil. In $12^{\circ}$ Congresso Brasileiro de Parasitologia Veterinária e $1^{\circ}$ Simpósio Latino-Americano de Rickettsioses. Ouro Preto, p. 333.

SMIT, F.G.A.M. 1987. An illustrated catalogue of the Rothschild Collection of fleas (Siphonaptera) in the British Museum (Natural History). Malacopsylloidea. Oxford University Press, Oxford \& London, v.7.

TIPTON, V.J. \& MÉNDEZ, E. 1966. The fleas (Siphonaptera) of Panama. In Ectoparasites of Panama (R.L. Wenzel \& V.J. Tipton, ed.). Field Museum of Natural History, Chicago, p.289-385.
TIPTON, V.J. \& MACHADO-ALLISON, C.E. 1972. Fleas of Venezuela. Brig. Young Univ. Sc. Bull. 17(6):1-115.

TRAUB, R. 1980. The zoogeography and evolution of some fleas, lice and mammals. In Fleas (R. Traub \& H. Starcke, ed.). A. A. Balkema, Rotterdam, p.93-172.

VAZ, Z. \& ROCHA, V.F. 1946. Tunga penetrans (L., 1758), "bicho do pé" em gado bovino. Importância clínica, observações parasitológicas e tratamento pelo D.D.T. Livro de homenagem a R.F. d'Almeida 40:327-332.

WENZEL, R.L. \& TIPTON, V.J. 1966. Some relationships between mammal hosts and their ectoparasites. In Ectoparasites of Panama (R.L. Wenzel \& V.J. Tipton, ed.). Field Museum of Natural History, Chicago, p.677-723.

Recebido em 06/07/2010

Versão reformulada recebida em 14/10/2010

Publicado em 15/12/2010 